\title{
Vers une relecture de l'univers onirique du discours imaginaire chez Kafka. Etude thématique et analytique
}

\author{
Dr. Kamel Mounir \\ Faculté des Lettres -Menoufeya
}

\section{Résumé}

Cet article a pour but de répondre à cette question : pourquoi Franz Kafka écrit-il ce qu'il écrit et quelle est la place de l'onirisme dans son univers romanesque ? Et à travers un paysage imaginaire qui porte des sens cachés ou métaphoriques nous aurons assez largement recours à la création de l'adjectif kafkaïen. Cela parait évidemment dans les quatorze récits du recueil "Un médecin de campagne" parut en 1919 écrit par Kafka en 1917 et projet d'étude présente. La première lecture de cette nouvelle nous montre clairement le caractère onirique du récit; les événements qui se déroulent dans la nuit sont plus proches des rêves que de la réalité. On peut même dire qu'ils sont nés du rêve. Dans ce texte les réflexions fabuleuses et légendaires exercent une influence permanente sur le lecteur. C'est aussi l'art de Kafka qui exprime ses conflits intérieurs et qui restent toujours le thème exclusif de sa littérature.

Mots clés : L'allégorie. Le fantastique. L'onirisme. La temporalité. L'animalité 


\section{نهو قراءة ثانية للعالَم الوهـهي في الخطاب الغيالي عند كافكا: دراسة هوضوواتية وتمليلية}

\section{د. كاهل همهمد هنيز همهد كاهل هوبسى \\ مدرس الأدب الفرنسي - كلية الآداب بالمنوفية - جامعة المنوفية همية همية}

تهدف هذه الورقة إلى الإجابة على السؤال التالي : لماذا يكتب فرانز كافكا هذا

العمل ويتخدم مشهر خيالي يحمل في طياته معاني ضمنية أو مجازية ـ يظهر هذا

الأمر بوضوح في مجموعته القصصية المؤلفة من أربعة عشر قصة قصيرة نذكر من بينها " طبيب في الريف " لفرانز كافكا، وهي القصة الثهيرة والتي تحمل نفس عنوان المجموعة القصصية الكاملة.

توضح لنا القراءة الأولى لهذه الرواية طبيعة العالم الوهمي الذي يشبه الحلم، ولهذا فان الأحداث التي تجري خلال الليل تعتبر أقرب إلى الحلم منها إلى الواقع • يمكننا القول بأن هذا الحلم يسمح للكاتب بإعادة التذكير بما حدث خلال حياته الماضية ـ في هذا النص الروائي، تعتبر التأملات الخرافية والأسطورية ذات تأثير مستمر على القارئ . ولهذا فان الفن الروائي عند كافكا يعبر عن الصراعات الداخلية عند الإنسان والتي تمثل الظاهرة الأدبية الأبرز عند كافكا. الكلمات المفتاحية: الحكاية الرمزية، الحكاية الخيالية، العالم الثبيه بالحلم، الزمانية، خصائص لا إنسانية. 
Vers une relecture de l'univers onirique du discours imaginaire chez Kafka. Etude thématique et analytique

Dr. Kamel Mounir

مجلة وادي النيل للاراسات والبحوث الإنسانية والاجتماعية والتربوية (مجلة علمية محكمة)

\section{Introduction}

Pour qualifier l'univers romanesque de Kafka (1883-1924) on se retrouve devant un choix qui marque un intérêt porté constamment au rêve par le romancier. Cela parait évidemment dans les quatorze récits du recueil "Un médecin de campagne" parut en 1919 écrit par Kafka en 1917 et projet d'étude présente. Ce recueil incarne d'une manière évidente un paysage imaginaire qui porte des sens cachés ou métaphoriques.

Claude David a remarqué " qu'il n'y a pas si longtemps que les récits de Kafka étaient encore traités comme des devinettes à déchiffrer. Comme on ne les comprenait pas, on leur prêtait des sens cachés, on y cherchait des significations allégorique, on en faisait des grimoires. ${ }^{(1)}$

La première lecture de cette nouvelle nous montre clairement le caractère onirique du récit; les événements qui se déroulent dans la nuit sont plus proches des rêves que de la réalité. On peut même dire qu'ils sont nés du rêve. Dans ce texte les réflexions fabuleuses et légendaires exercent une influence permanente sur le lecteur. C'est aussi l'art de Kafka qui exprime ses conflits intérieurs et qui restent toujours le thème exclusif de sa littérature.

Ajoutons que la sexualité trahie est pareillement un thème fantastique avec l'impuissance de la vocation: comment le médecin (Kafka) veut sauver un malade lorsqu'il souffre de la même maladie. Tout cela amène la réflexion de l'auteur vers la monotonie du quotidien à tel point que le médecin (l'écrivain) voit le présent comme une chose invisible et impitoyable.

1- David (Claude), Préface et notes sur Un artiste de la faim et autres récits. Franz Kafka.Ed.Gallimard, 1980.P7 
(ISSN : 2536 - 9555)

C'est donc à travers cela que l'on peut dégager les trois thèmes essentiels du récit.

- L'image onirique du médecin.

- Le rapport entre le médecin et la collectivité.

- L'importance du temps et des lieux.

En premier lieu, nous allons aborder le thème des images oniriques qui évoquent des obsessions sexuelles du médecin à travers les autres personnages, les animaux, les objets et les différents lieux du récit.

Les rapports entre le médecin et la collectivité constituent un point sur lequel il est intéressant de se pencher, il sera donc l'objet de notre seconde partie.

Enfin nous ne manquerons pas de mettre en évidence l'intérêt du temps et des lieux ainsi que leur influence sur les éléments et les personnages dans la nouvelle.

\section{Les arrières pensées avec lesquelles tu accueilles en toi le mal ne sont pas les tiennes mais celles du mal.}

\section{Franz Kafka}

Franz Kafka, l'écrivain thèque d'expression allemande, est l'un des auteurs les plus étudiés de la littérature du xx siècle. Le jeune Kafka fut marqué par un triple héritage culturel : il découvrit la Bible et la langue hébraïque à travers l'héritage juif. De l'héritage thèque il lut la littérature russe thèque et polonaise.

En plus, il fit toutes ses études en allemand bénéficiant de la culture germanique. Apres des études de droit, Kafka occupa des postes différentes mais l'écriture reste pour lui une passion permanente. L'univers irréel, la culpabilité, la perte d'identité et la transformation du corps représentent les thèmes essentiels dans l'œuvre de Franz Kafka. 
Vers une relecture de l'univers onirique du discours imaginaire chez Kafka. Etude thématique et analytique

Dr. Kamel Mounir

مجلة وادي النيل للاراسات والبحوث الإنسانية والاجتماعية والتربوية (مجلة علمية محكمة)

Marc Goldschmit a bien constaté que " Il faut plutôt chercher comment l'écriture de Kafka joue de la lettre et de l'allégorie, de l'allégorie de la lettre et de la lettre de l'allégorie" ${ }^{\prime(2)}$

Le récit un médecin de champagne est appelle a visiter un patient dans village n'est très loin de sa maison. Mais malheureusement son cheval vient de mourir. Le malade est jeune qui accueille le médecin en disant: "Docteur, laisse-moi mourir" Le médecin examine le garçon mais en même temps il pense a Rose sa bonne.

Le médecin pense aussi à sa misère et se dit à lui-même qu'il veut mourir et au moment où il va partir il découvre que le garçon souffre d'une plaie. Le médecin se trouve lui-même dans le lit du malade. Malheureusement, tout va mal le garçon meurt et le médecin n'arrive pas à le sauver. En plus, les cheveux sont mal attachés l'un a l'autre.

Les images oniriques du médecin, ce sera l'objet du premier chapitre. Au terme de ce parcours, on espère ainsi avoir avec l'aide d'une lecture approfondie à illustrer un médecin qui est réveillé au milieu de la nuit pour soigner un patient. Le médecin ne voit rien, ensuite il découvre une mauvaise plaie sur le flanc du malade :

" Dans la chambre du malade, l'air est à peine respirable; le poêle, dont on a négligé de s'occuper, s'est a mis fumer ; je vais devoir ouvrir la fenêtre, mais je veux d'abord voir le malade. Maigre, sans fièvre, ni froid ni chaud, les yeux vides, sans chemise, le jeune garçon se relève sous l'édredon, se prend à mon cou, me murmure à l'oreille : Docteur, laisse-moi mourir" (3)

2 - Marc Goldschmit. Kafka, la faim de l'écriture et l'animalittérature. Les cahiers philosophiques. P.267.2013.

https://doi.org/10.4000/cps.1949.

3 - Franz Kafka.Un médecin de champagne. Ed.Gallimard 1980.P.113 
(ISSN : 2536 - 9555)

En premier lieu, le patient voulait cesser de vivre et quitter ce monde, le médecin de son côté se doute sur la façon de traiter son patient ou de s'échapper: " C'est seulement maintenant que je repense à Rode ; que faire? Comment la sauver ? Comment la tirer des mains de ce valet d'écurie, quand les cheveux attelés à moi voiture refusent de m'obéir" (4)

Dans une langue sobre et minutieuse, Kafka a tenté d'éclairer la vie familière faisant pénétrer le lecteur dans une existence tourmentée dans laquelle se succèdent les échecs. C'est la raison pour laquelle l'œuvre de Kafka ne cesse de fasciner les psychanalystes. A travers ses deux romans majeurs : 'Le Procès' et 'Le Château', il y développe avec angoisse et ironie un univers absurde, un monde que le langage courant qualifiera par la suite de 'kafkaïen'.

Nous remarquons que la métamorphose est effectuée dès la première phrase de récit et que le lecteur reste dans le doute en ce qui concerne la culpabilité du héros. On oscille entre le monde réel et la métamorphose.

C'est la raison pour laquelle nous pouvons affirmer que Le monde pour Kafka est une métaphore. Métaphore de qui, de quoi ? Là est toute la question, que notre romancier laisse volontairement sans réponse.

"L'importance du rêve, dans le Journal et dans le processus créateur kafkaïen, n'est plus à prouver. Kafka lui-même a défini le rêve comme fondement de son écriture en déclarant que toute son existence se réduisait à sa volonté de décrire sa "vie intérieure, qui a quelque chose d'onirique ». Le rôle du rêve chez Kafka est attesté par le seul nombre de récits de rêves du Journal - une trentaine environ." ${ }^{(5)}$

4 - Franz Kafka op:cit, P.16

5 -Le Journal de Kafka, atelier de l'écrivain.

http://www.openedition.org/6540. Chapitre IV. Du procès du rêve au processus onirique.Ed.2001.P.317 
Vers une relecture de l'univers onirique du discours imaginaire chez Kafka. Etude thématique et analytique

Dr. Kamel Mounir

مجلة وادي النيل للاراسات والبحوث الإنسانية والاجتماعية والتربوية (مجلة علمية محكمة)

Cela souligne que l'écriture onirique de Kafka est témoignage sur ses rapports avec lui-même et avec l'univers qui l'entoure.

\section{Le valet et la servante} en disant :

L'auteur nous donne une idée de l'aspect physique du valet

"Un homme accroupi dans l'étroit réduit montrait son visage aux yeux bleus pleins de franchise" (Kafka.1980.p.111)

A travers cette phrase il suggère la sensualité, celle-ci est toujours présente dans les récits de Kafka où l'image onirique de la sexualité a une place importante.

Maurice Blanchot de sa part voit que " la lecture symbolique est probablement la pire façon de lire in texte" (6)

Le valet, dont l'existence était inconnue du médecin semble doux et calme au premier abord mais en un instant sa douceur disparait, son attitude change notamment avec la servante.

Il se jette sur elle et la mort au visage sans aucune raison. Cette brutalité apparaît comme une pulsion sexuelle du valet, mais elle peut aussi être vue comme une pulsion du médecin.

Le valet incarne ici la sexualité et l'instinct. Il agit de manière incompréhensible, comme poussé par une force intérieure qui pourrait être les fantasmes sexuels du médecin envers la servante.

Celui-ci rejette le coté brutal, bestial de ses fantasmes qu'il ne peut accomplir puisqu'il les rejette. Ainsi c'est le valet qui agit tel que lui aurait aimé agir.

$\left({ }^{6}\right)$ - M. Blanchot, le livre à venir, Paris.Gallimard, 1959.P.105 
(ISSN : 2536 - 9555)

Ce récit étant onirique, on pourrait imaginer que les faits n'ont pas réellement existés, c'est-à-dire que le valet lui-même est né de l'imagination du médecin. Celui-ci se sert, en quelque sorte du valet pour extérioriser ce qu'il ressent.

La phrase "Comme s'il devinait mes pensées" (Kafka.1980P.112) nous montre clairement que le valet et le médecin sont en parfait accord, comme si ces deux personnages dans le récit n'étaient en fait qu'une seule personne.

De ceci, nous pouvons tirer deux interprétations possibles :

- La première est que le valet est, en fait, la réalisation des fantasmes du médecin. C'est un personnage imaginaire inventé par le médecin pour pouvoir faire réaliser ses désirs sexuels par quelqu'un d'autre que lui. Kafka pouvait être identifié par le médecin tandis que le valet serait alors l'esprit torture du médecin et donc de Kafka lui-même.

A l'opposé de la remarque de Maurice Blanchot, nous constatons que Hermann Broch affirme que " Si l'homme ne possédait pas le rêve ouvert sur le réel, il se tiendrait sans langage comme l'animal, en présence des phénomènes du réel, mais comme il possède ce rêve et avec lui le domaine symbolique du langage, celui-ci devenu une seconde réalité."(7)

La deuxième interprétation est que l'on peut voir dans l'existence des deux personnages ; le médecin et le valet, deux aspects de la personnalité de Kafka. Le valet représentant ses fantasmes, le mal, le mauvais côté de sa personnalité et en plus sa maladie.

Et le médecin, de sa part, représente le bon côté, sa morale. Ceci montrerait le conflit qui existe dans l'esprit de Kafka entre ses pulsions et son éducation: " D'une manière plus générale, les récits de Kafka ne frappent ni par l'élégance du style, toujours aussi plat, ni par l'intérêt des histoires, originales mais

7 - Hermann Broch, Création littéraire et connaissance. Gallimard, 1966.P.142 
Vers une relecture de l'univers onirique du discours imaginaire chez Kafka. Etude thématique et analytique

Dr. Kamel Mounir

مجلة وادي النيل للاراسات والبحوث الإنسانية والاجتماعية والتربوية (مجلة علمية محكمة)

indéchiffrables. Non, ce qui frappe justement chez Kafka et qui rend sa lecture incontournable c'est justement cette démonstration qu'il n'y a pas d'histoires."(Kafka.1980p.110)

\section{L'écurie}

Nous remarquons que l'image de la sexualité est aussi suggérée par l'écurie. Cela parait évident dans la citation suivante :

" Elle s'ouvrit, en allant et venant sur ses gonds. Une chaleur et une odeur de chevaux émanaient de l'endroit. Une lanterne sourde d'écurie se balançait au bout d'une corde." (Kafka.1980p.111)

Il est facile d'interpréter le mouvement de la porte et de la lanterne, de même que la chaleur et l'odeur qui se dégage de cet endroit.

En effet, les mouvements de va et vient de la porte et de la lanterne peuvent apparaître comme une invitation, un appel lancé au médecin pour qu'il entre dans l'écurie. Ce sont ces éléments qui font naître les pulsions, les envies sexuelles du médecin car il les ressent comme une demande, comme un appel à lui de le part d'une femme.

De même, la chaleur et l'odeur qui se dégage de ce lieu ne font que renforcer cette invitation et le médecin ne peut résister à cet appel, son instinct sexuel le pousse à entrer, à laisser libre cours à ses envies, à ses désirs qui sont sexuels.

C'est à ce moment-là, où son envie est très grande qu'apparaît le valet dans le récit. Celui-ci se trouve dans un endroit très étroit d'où il doit ramper pour sortir. Il est dans une place très petite dans le récit, place qui va grandir par la suite.

On peut penser que le médecin voit alors ce valet comme une personne qui peut lui faire oublier ses envies. D'ailleurs, 
(ISSN : 2536 - 9555)

l'apparition du valet va permettre au médecin de se libérer de ses envies en les rejettent sur le valet.

\section{Les deux chevaux}

Les chevaux de Kafka jouent un rôle essentiel dans les évènements du récit comme phénomènes psychiques face à l'homme. Cette confrontation à l'animalité évoque également une image métaphorique dont les éléments sont cohérents et qui représentent avec précision une idée allégorique sur les deux cheveux.

"L'animal occupe une grande place dans la culture, et il sera intéressant de voir parallèlement comment des ouvres artistiques (littéraires et picturales) éclairent à leur manière l'articulation entre l'humain et l'animal. Les artistes dévoilent la part de l'animalité en nous, aux limites de l'humain, avec la figure d'animal dans certaines œuvres d'art remarquables : le chien de Goya, le chien de Miro, les chevaux de Franz Marc." ${ }^{\prime(8)}$

Simone Korff-Sausse a mis en relief la part animale dans le sujet humain en affirmant que " Dans le champ de la psychanalyse, l'animal fait des occurrences très nombreuses : le rêve de l'Homme aux loups, l'Homme aux rats, le vautour de Léonard de Vinci, le cheval du petit Hans, les coqs du petit Arpad de Ferenczi. Dans toute son cuvre, Freud a révélé la part animale dans le sujet humain, en faisant une équivalence entre inconscient et animalité" $^{(9)}$

"Holà garçon holà, la fille" (Kafka.1980.p. 111)

$\mathrm{Ce}$ cri de palefrenier montre que les deux chevaux sont de sexe opposé, la précision apportée ici prouve le caractère sexuel de ce passage.

8 - Simone Korff-Sausse. Les identifications déshumanisantes : l'animalité dans la vie psychique et la création artistique. Revue française de psychanalyse. 2011/1 Vol.75 p.88

9 - Simone Korff-Sausse. Les identifications déshumanisantes.op.cit.p.89 
Vers une relecture de l'univers onirique du discours imaginaire chez Kafka. Etude thématique et analytique

Dr. Kamel Mounir

مجلة وادي النيل للاراسات والبحوث الإنسانية والاجتماعية والتربوية (مجلة علمية محكمة)

Le médecin ne manque pas de remarquer la puissance. La force des chevaux, il souligne leurs reins puissants, la forme harmonieuse de leurs têtes. Les deux bêtes représentent la beauté, la force et la puissance de l'amour, de la sexualité.

Leur attitude lorsqu'elles franchissent la porte de l'écurie " L'une derrière l'autre" "Par de puissants coups de croupe" et une fois dehors lors qu'elles se redressent sur leurs pattes, montre l'envie, le désir sexuel du médecin.

Cela veut dire, que si l'on interprète le fait, que les deux bêtes se redressent sur leurs pattes comme un signe de victoire de libération, et ceci toujours par rapport aux obsessions sexuelles on peut penser qu'en sortant de l'écurie qui est nous l'avons dit le symbole du sexe féminin.

Elles sont heureux, libérées, et le médecin voudrait ressentir lui aussi cette joie et cette libération après avoir agir selon ses fantasmes.

D'ailleurs, cette scène peut être comparée à un accouchement. Les bêtes donnent des coups dans la porte pour pouvoir sortir, puis elles sortent l'une derrière l'autre, les pattes collées au corps et elle occupe l'ouverture de la porte toute entière, et "le corps enveloppé d'une épaisse vapeur" (Kafka.1980.p. 112)

C'est la raison pour laquelle la scène où le valet mord la servante au visage est situé juste après celle des chevaux enveloppées de vapeur car le médecin laisse le valet agir selon ses fantasmes pour pouvoir ressentir le bien être qu'il attend.

\section{La plaie du médecin :-}

Le médecin nous donne une description détaillée de la blessure du malade, nous constatons cela à la page 112 "Une blessure s'est ouverte, grande comme la paume" ceci pour nous montrer l'importance de cette blessure à ses yeux. Celle - ci est horrible, remplie de vers et immaculée de sang. 
(ISSN : 2536 - 9555)

Si l'on reste dans la perspective des obsessions sexuelles du médecin, cette blessure peut apparaître elle encore comme le sexe de la femme. Elle est le résultat d'un coup de pioche, outil qui pourrait lui être rapproche du sexe masculin.

D'ailleurs, la description même de la blessure revoit à la servante Rose. "Cette jolie fille qui vit chez moi depuis des années, sans que j'aie même pris garde à elle."(Kafka.1980.p.115)

À la Page 117 nous remarquons une autre citation :

"Il en est beaucoup ..."

D'une part, cette citation met en évidence que le médecin veut dire qu'il existe des femme qui s'offrent aux hommes sans le vouloir, d'autre part, les vers sortant de la blessure montrent l'horreur de celle-ci et donc le caractère sale ou impur du sexe féminin tel que le médecin le voit.

Cette blessure va conduire la maladie à la mort comme si la sexualité du médecin ou plutôt ses fantasme vont le conduire lui aussi à la mort. Il prend conscience de sa maladie et voudrait lui aussi mourir.

\section{Médecin et collectivité}

Ce thème nous donnera l'occasion de rendre plus clair le rapport entre le médecin et la collectivité constituent un point sur lequel il est intéressant de se pencher. Ce qui affirme ce concept c'est la parole de Jean-Paul Sartre dans Qu'est-ce que la littérature?:

"La collectivité passe à la réflexion et à la médiation, elle acquiert une conscience malheureuse, une image sans équilibre d'ellemême, qu'elle cherche sans cesse à améliorer" (10)

Le médecin et sa servante :-

Tout au long du récit nous voyons que le médecin accorde à sa servante une place particulière. D'une part parce qu'il vit tout

10 - Jean-Paul Sartre. Qu'est-ce que la littérature ? Gallimard.1947.p.29 
Vers une relecture de l'univers onirique du discours imaginaire chez Kafka. Etude thématique et analytique

Dr. Kamel Mounir

مجلة وادي النيل للاراسات والبحوث الإنسانية والاجتماعية والتربوية (مجلة علمية محكمة)

seul souffre de l'ennui et de la solitude, d'autre part parce qu'elle seule s'occupe de lui. Par exemple lorsque le médecin perd son cheval, sa servante se propose d'aller en cher un autre au village.

Alors que le médecin la croyait au village, elle est la près de lui avec sa lanterne.

$\mathrm{Au}$ moment où la servante doit apporter son aide au médecin c'est elle qui semble avoir besoin de lui cela parait évident quand le valet se jette sur elle et la mort au visage "Elle crie et cherche refuge auprès de moi" (Kafka.1980.p.116)

Le médecin ne l'interdit pas et il se contente d'être agressif envers le valet en le traitant d'animal.

Lorsque rose crie pour la deuxième fois craignant le valet qui reste auprès d'elle, elle ne trouve aucune réaction de la part du médecin. Il en est de même lorsque il part abandonnant rose malgré ses cris et le tumulte dans la maison.

En revanche, dans la maison du malade, il ne cesse pas de repenser à sa servante, elle est toujours dans sa tête il ne sait pas comment il peut la sauver des mains du valet.

Nans remarquons un conflit entre le bon, et le mauvais qui se déroule dans la tête, ce conflit l'éloigne de ceux qui l'entoure, ce conflit a eu une mauvaise influence sur son métier, son devoir envers rose le fait ressentir des regrets et des remords à tel point qu'il préfère mourir comme son malade. La mort pour lui est beaucoup mieux que de faire du mal à rose.

L'obsession sexuelle dont nous avons parlé dans la première partie est la raison pour laquelle le médecin préfère mourir que de rentrer chez lui, non pas pour protéger sa servante du valet, mais pour la sauver de lui-même.

Ce qui affirme cette idée est les citations suivantes " Il faut encore que je m'occupe de Rose.... (Kafka.1980.p. 114). 
(ISSN : 2536 - 9555)

Nous constatons que dans toutes les pages du récit le médecin ne pense pas à son malade autant qu'a Rose. Il ne s'inquiète pas de ne pas pouvoir le guérir, il ne pense qu'à la protection de sa servante. Il prête à Rose une importance plus grande que celle du malade. Il voit que le gamin se porte bien tandis que sa servante se porte mal. Il trouve qu'elle a besoin de lui beaucoup plus que.

De plus, le médecin tente d'expliquer qu'il s'occupe de d'elle apparemment parce qu'elle est une jolie fille et elle vit chez lui depuis des années. C'est - à- dire qu'il pense qu'il est temps pour lui de prendre soin d'elle.

En fin du récit, le médecin n'a aucune envie de rentres chez lui, il renonce à rentrer, certainement parce qu'il n'a pu sauver Rose et surtout parce qu'il sait qu'il ne paner pas la protéger de luimême, car c'est lui qui lui veut du mal par ses fantasmes, ce n'est pas le valet.

Bien qu'au fond de lui il ne souhaite que l'aider, il ne fait rien pour elle et c'est cela qui lui fait perdre l'espoir. Le rapport entre le médecin et le malade souligne que le titre du récit nous montre justement que le médecin et la compagne forment les deux pôles des évènements.

Rose la servante du médecin vient en premier lieu car elle a comme nous l'avons dit, une place importante dans sa pensée. Il parle du malade dans la deuxième ligne de la nouvelle en disant " un grand malade m'attendait" puis maison du malade il parle de Rose des chevaux du valet jusqu'au moment où il arrive à la.

Le médecin ne prête pas beaucoup d'attention au malade. Celui-ci non plus n'attend pas grande chose du médecin. Tandis que ses parents lui demandent l'impossible pour sauver leur fils. Les parents du malade représentent, en fait, tous les villageois de la compagne qui attendent du médecin qu'il fasse le maximum.

Le médecin pour les villageois doit être un sorte de sauveur, son rôle est de guérir les gens en faisant tout ce qui est possible El le fait de le considérer comme un sauveur, attendant même des 
Vers une relecture de l'univers onirique du discours imaginaire chez Kafka. Etude thématique et analytique

Dr. Kamel Mounir

مجلة وادي النيل للاراسات والبحوث الإنسانية والاجتماعية والتربوية (مجلة علمية محكمة)

miracles de sa part, lui donne d'un prêtre. Mais le médecin refuse ce rôle car s'il était prêtre, il pouvait remettre à Dieu le sort de rose. Il est convaincu qu'il ne peut reformer le monde il n'arrive pas à protéger sa servante alors comment protégeait- il le monde.

\section{L'importance du temps et des lieux}

Il nous reste pour préciser le cadre spatio-temporel la question de référence entre temps et lieu. Paul Ricœur a remarqué que " Le monde déployé par toute ouvre narrative est toujours un monde temporel : le temps devient temps humain dans la mesure où il est articulé de manière narrative ; en retours le récit significatif dans la mesure où il dessine les traits de l'expérience temporelle." $(11)$

Le beau et le mauvais temps :

Tout au long de nouvelle existe une forte opposition entre le beau et le mauvais temps qui révèle, en fait, les états d'âmes du médecin. Dès la première ligne apparaît la relation entre le mauvais temps et l'humeur du médecin, nous voyons cela nettement à la page (Kafka.1980.p.118).

" J'étais dans un vif embarras : une forte tempête de neige emplissait le vaste qui me déparait de lui"

Ajoutons que la mort de son cheval dans cet hiver glacial est la raison pour laquelle il est aussi dans un vif embarras. Par ailleurs, l'ennui et la solitude qui le caractérise sont accentués par l'hiver qualifie de glacial et plus précisément par la tempête de neige.

Le fait du froid et de la glace est très remarquable dans l'histoire en effet, le médecin désespère à la sensation que le temps se fige qu'il ne peut lutter contre lui lui-même est immobile dans l'impossibilité de bouger.

11 - Paul Ricœur. Temps et récit. Tome I. Edition du Seuil.1983.P.17 
(ISSN : 2536 - 9555)

Sans parler de fixation du temps, on peut parles d'accélération par exemple entre le moment où le médecin quitte sa maison et moment où il arrive chez le malade, il semble s'écoule qu'un court instant.

"Comme si la ferme de mon malade se trouvait à la porte de chez-moi" (Kafka.1980p.119)

L'autre rapport avec le temps est la lumière qu'elle entrevoit d'abord à travers la lanterne de la servante, puis à travers la porte de l'écurie. Cette lumière qui se dégage près que simultanément de la lanterne et de l'écurie est en fait le regain d'espoir que ressent alors le médecin.

Cette chaleur qui l'allaise et qui est en contraste avec le froid externes la lanterne et notamment sa lumière est le signe de l'espoir. C'est ce qu'attendait le médecin,

Plus tard dans le récit un clair de lune apparaît. Ce clair de lune montre le calme, la tranquillité ressentis par le médecin. Alors qu'il se trouve devant la porte de son malade, la neige cesse de tomber là encore c'est le signe de la tranquillité.

Les lieux ouverts et les lieux clos :

Il existe aussi une forte opposition entre les lieux ouvert et les lieux fermés (clos). La succession de lieux au cours du récit est sont aussi le reflet de l'humeur du médecin (narrateur).

$\mathrm{Au}$ début du récit l'espace est décrit comme vaste, ce cil montre la grandeur du désarroi du narrateur. Il est seul dans cet espace qui semble immense et contre lequel il semble désemparé, ne pouvant rejoindre son malade de même qu'en attend un regard sur lui.

L'écurie $1^{\mathrm{er}}$ lieu fermé du récit, est étroit " était réduit" la maison, autre lieu fermé est pu le médecin un lieu d'embarras de solitude alors que celle- ci est un refuge pour la servante qui fuit le valet.

Plus tard lorsqu'il entre dans la maison de son malade, il se sent étouffer par l'atmosphère il se trouve dans lieu ferme comme 
Vers une relecture de l'univers onirique du discours imaginaire chez Kafka. Etude thématique et analytique

Dr. Kamel Mounir

مجلة وادي النيل للاراسات والبحوث الإنسانية والاجتماعية والتربوية (مجلة علمية محكمة)

s'il était emprisonne ne pouvant fuis l'air est irrespirable cette atmosphère, lourd montre la tentions qui règne dans la maison et plus précisément chez le médecin.

\section{Conclusion}

Kafka dans cette nouvelle nous montre la faiblesse et la misère de son héros. L'atmosphère onirique qui domine dans tout le récit a pour but de révéler l'état psychique de Kafka qui s'identifie au médecin.

Cette nouvelle raconte de manière détournée le conflit qui existe dans l'esprit de l'auteur. Ce conflit étant principalement lie aux obsessions sexuelles de l'auteur qu'il tente d'expliques á travers les personnages les animaux, les objets, et les différents lieux du récit le temps lui a pour but de montrer á travers les accélérations et le ralentissement que l'auteur ne peut maitrises ses obsessions.

Kafka insiste également dans tous ses écrits sur le rapport entre l'homme et le monde laissant derrière lui une œuvre inachevée mais monumentale. D'une manière plus générale, les récits de Kafka ne frappent ni par l'élégance du style, toujours aussi plat, ni par l'intérêt des histoires, originales mais indéchiffrables. Non, ce qui frappe justement chez Kafka et qui rend sa lecture incontournable c'est justement cette démonstration qu'il n'y a pas d'histoires. 
مجلة و ادي النيل للاراسات والبحوث الإنسانية والاجتماعية والتربوية (مجلة علمية محكمة)

(ISSN : 2536 - 9555)

\section{RÉFÉRENCES BIBLIOGRAPHIQUES}

\section{Corpus}

Franz KAFKA, (1952), Un médecin de campagne, trad. Alexandre Vialatte.

\section{OUVRAGES}

Jean-Paul Sartre. Qu'est-ce que la littérature ? Gallimard.1947.

Arnaud VILLANI, (1984), Kafka : l'ouverture de l'existant, Paris, Belin.

Claude THIEBAUT, (1996), les Métamorphoses de Franz Kafka, Paris, Gallimard

David CLAUDE, (1980), Franz Kafka, Paris, Gallimard.

Ernst PAWEL, (1988), Franz Kafka ou le cauchemar de la raison, Paris, Le Seuil.

Franz KAFKA, (1980), Un artiste de la faim et autres récits. Texte intégral, Gallimard.

Franz KAFKA, (1980), Euvres complètes, t. II, Paris, Gallimard, Bibl. de la Pléiade.

Franz KAFKA, (1988), Euvres complètes, 4 vol. trad. Marthe RoBERT, Davide CLAUDE et Jean-Pierre DANÈS, édition de Claude David, Paris, Gallimard, coll. "Bibliothèque de la Pléiade".

Franz KAFKA, (1994), Journal, trad. M. Robert, Paris, Grasset.

Franz KAFKA, (1995), Lettre au père, trad. M. Robert, Paris, Gallimard (Folio bilingue).

Gilles DELEUZE et Félix GUATTARI, (1975), Kafka, pour une littérature mineure, Paris, Minuit. 
Vers une relecture de l'univers onirique du discours imaginaire chez Kafka. Etude thématique et analytique

Dr. Kamel Mounir

مجلة وادي النيل للاراسات والبحوث الإنسانية والاجتماعية والتربوية (مجلة علمية محكمة)

Hermann BROCH, (1966), création littéraire et connaissance, trad. Albert Kohn. Édition d'Hannah Arendt. Introduction d'Hannah Arendt, Gallimard.

Jean MONTALBERTI, (1983), Kafka le Pragois, in Le Magazine littéraire, $\mathrm{n}^{\circ} 198$.

Jean. FLORENCE, (1985), Le désir de la loi, in J. FLORENCE, Ouvertures psychanalytiques, Bruxelles, Publications des Facultés universitaires Saint-Louis.

Jean-Pierre MOREL, (1998), Le Procès de Franz Kafka, Paris, Gallimard.

Marthe. ROBERT, (1979), Seul comme Franz Kafka, Paris, Calmann-Lévy.

MAURICE BLANCHOT, (1981), De Kofka à Kafka, Paris, Gallimard (Folio).

Paul RICOEUR, (1983), Temps et Récit L'Intrigue et le Récit historique, Paul Ricœur, Paris, Seuil,

Paul RICOEUR, (1990), Soi-même comme un autre, Paris, Seuil.

Paul RICOEUR, (1991), Éthique et morale, in Lectures 1, Paris, Seuil.

Paul RICOEUR, (1995), Qui est le sujet de droit?, in Le juste, Paris, Esprit.

Paul ROBERT, (1970), Dictionnaire alphabétique et analogique de la langue française, Paris, Société du nouveau Littré, t. III.

Pierre CAMINADE, (1970), image et métaphore, Paris, édition bordas.

Pierre MERTENS, (1996), Kafka écrivain « engagé » [PDF], Bruxelles, 
مجلة وادي النيل للاراسات والبحوث الإنسانية والاجتماعية والتربوية (مجلة علمية محكمة)

(ISSN : 2536 - 9555)

Yasha DAVID et Jean-Pierre MOREL (1984), le Siècle de Kafka, collectif, Paris, Centre Georges Pompidou.

\section{WEBOGRAPHIE}

https://doi.org/10.4000/cps.1949.

https://books.openedition.org/pusl/8429

Marc Goldschmit, «Kafka, la faim de l'écriture et l'animalittérature », Les Cahiers philosophiques de Strasbourg [En ligne], 33|2013, mis en ligne le 15 mai 2019, consulté le 04 octobre2021. URL :

http://journals.openedition.org/cps/1949; DOI :

https://doi.org/10.4000/cps.1949- 